\title{
Evolutionary status of early-type galaxies in distant poor clusters
}

\author{
Alexander Fritz and Bodo L. Ziegler \\ ${ }^{1}$ Universitäts-Sternwarte Göttingen, Geismarlandstraße 11, 37083 Göttingen, Germany email: \\ afritz@uni-sw.gwdg.de
}

\begin{abstract}
We introduce our project that investigates the kinematic properties of early-type galaxies in 6 distant poor clusters at $z \approx 0.25$. This study represents a continuation of our efforts to understand galaxy evolution in low-density environments. Higher-resolution MOSCA spectra have been obtained at the Calar Alto 3.5-m telescope with which we can measure absorption line strengths and velocity dispersions. In conjunction with our $H S T / F^{\text {r }} 02 \mathrm{~W}$ images of all the clusters, we are able to construct the Fundamental Plane of ellipticals and S0 galaxies in poor clusters at a look-back time of $\approx 3$ Gyr. For galaxies outside the HST field, we concentrate our analysis on the $\mathrm{Mg}-\sigma$ and Faber-Jackson relations. With the line strength diagrams age/metallicity distributions can be derived in densities between the field and rich cluster environments. Comparing with our rich clusters at the same cosmic epochs, the dependence of galaxy formation models on the local environment can be tested more quantitatively.
\end{abstract}

\section{Introduction}

Recent analyses of the kinematics and stellar populations of faint distant galaxies up to redshifts of $z \approx 1.0$ have put strong observational constraints on galaxy formation models. The weak evolution seen in the tight correlation of the distance independent parameters $\mathrm{Mg}$-absorption and velocity dispersion, $\sigma$, at $z \approx 0.4$ is evidence for a high redshift $\left(2<z_{f}<4\right)$ of formation for the bulk of the stars in cluster ellipticals (e.g., Ziegler \& Bender 1997). Further support gives the small increase of the $B$-band luminosity derived from the Faber-Jackson relation $(L$ vs. $\sigma$ ). The combination of structural (size and surface brightness) and kinematic $(\sigma)$ parameters in the Fundamental Plane (Djorgovski \& Davis 1987 ) allows us to investigate not only the luminosity but also the mass evolution of earlytype galaxies. Most previous studies of the Fundamental Plane (FP) at $0.2<z \leqslant 1.3$ revealed that the mass-to-light ratio of luminous galaxies evolves only mild in accordance with passive evolution models (Ziegler et al. 2001; van Dokkum \& Stanford 2003; see also contribution by Fritz \& Ziegler in this volume). However, these observational results apply only to the special environment of dense rich clusters.

Semi-analytic CDM models predict a hierarchical assembly for galaxies through merging of subunits and replenishment of gas from their halos (Cole et al. 2000). Only in the richest clusters galaxies experienced their last major merger at $z \sim 1-2$. Thus, the population of early-type galaxies should be more diverse in lower density regions.

For this reason, we analyse the evolutionary status of early-type $(\mathrm{E}+\mathrm{S} 0)$ galaxies in poor clusters at a look-back time of $\approx 3 \mathrm{Gyr}$ with the same quantitative methods as was done in rich clusters. The $\mathrm{Mg}-\sigma$ relation will reveal whether there is more spread in the age/metallicity distribution than in rich clusters at similar epochs, e.g., in Abell 2218 (Ziegler et al. 2001) or Abell 2390 (Fritz et al. 2004). Results on the FP will give insights into whether the mass/structure evolution is more consistent with the monolithic or the hierarchical formation scenario. We use $\Omega_{m}=0.3, \Omega_{\Lambda}=0.7$ and $h=0.7$. 
Table 1. Sample of six poor clusters.

\begin{tabular}{|c|c|c|c|c|c|c|}
\hline Cluster & $\begin{array}{l}\text { R.A. } \\
\qquad \text { (J2 }\end{array}$ & $\begin{array}{l}\text { Dec. } \\
000)^{-}\end{array}$ & $<z>$ & $\begin{array}{l}L_{\mathrm{X}}(0.1-2.4 \mathrm{keV}) \\
10^{43} h_{50}^{-2} \mathrm{ergs} \mathrm{s}^{-1}\end{array}$ & $N_{\text {obj } / \text { mask }}$ & $\begin{array}{l}T_{\exp } \\
(\mathrm{ksec})\end{array}$ \\
\hline $\mathrm{Cl} 0818+56$ & 081904 & +565449 & 0.2670 & 1.50 & 14 & 29.40 \\
\hline $\mathrm{Cl} 0841+70$ & 084144 & +704653 & 0.2397 & 1.22 & 13 & 24.54 \\
\hline $\mathrm{Cl} 0849+37$ & 084911 & +373109 & 0.2343 & 1.93 & 17 & 18.00 \\
\hline Cl 1633+57 & 163342 & +57 1412 & 0.2402 & 0.49 & 14 & 30.97 \\
\hline Cl 1701+64 & 170147 & +642057 & 0.2458 & 0.40 & 18 & 27.90 \\
\hline $\mathrm{Cl} 1702+64$ & 170214 & +64 1953 & 0.2233 & 0.74 & 18 & 27.90 \\
\hline
\end{tabular}

\section{The low- $L_{X}$ project}

This investigation is an intensive observational campaign to study the evolutionary status of galaxies in 10 poor clusters at intermediate redshifts of $0.2<z<0.3$ selected to have very low X-ray luminosities $\left(L_{X}<5 \times 10^{43} \mathrm{erg} / \mathrm{s}\right), 100$ times lower than the CNOC clusters, and poor optical richness class. It comprises ground-based optical and NIR photometry, medium-resolution multi-object spectroscopy, and $H S T / F 702 W$ imaging.

The clusters are dominated by passive galaxies with no or little on-going star formation (SF). The fraction of emission-line galaxies (ELG) is as low as in the rich X-ray bright CNOC clusters at similar epochs $\left(f_{\mathrm{ELG}} \approx 0.2\right)$ and at variance with the field value $\left(f_{\mathrm{ELG}} \approx 0.6\right)$. This indicates that processes heavily depending on density like rampressure stripping can not be the dominant mechanisms responsible for the supression of SF (Balogh et al. 2002b). Analysis of the HST images for all ten clusters revealed that the overall properties of these cluster galaxies are similar to those in more massive clusters with a low fraction of disk galaxies (Balogh et al. 2002a).

\section{Sample selection for spectroscopy}

The spectroscopic observations have been gained during eleven nights (04/2001 and 02/2002) with the MOSCA spectrograph at the Calar Alto Observatory (Spain). A summary of the cluster sample with the mean redshifts, number of objects on a mask and total exposure times for spectroscopy is given in Tab. 1. Early-type cluster members have been selected based on our low-resolution spectra and from their HST morphology. For the FP study, we obtained spectra of 5-7 galaxies lying within the HST fields in a single MOS mask. Five masks (one per cluster; Cl1701 and Cl 1702 only observed with one mask) have been observed to construct a combined FP sample of $\sim 30$ galaxies, comparable to that of a single rich cluster (Fritz et al. 2004). Outside the HST field additional spectra of $\sim 10 \mathrm{E}+\mathrm{S} 0$ in each cluster serve for the investigation of the $\mathrm{Mg}-\sigma$ and FaberJackson relation. For these scaling relations the number of observed galaxies is sufficient to explore possible variations between the different poor clusters as a function of their $\sigma$ or $L_{X}$. Our total sample comprises $\sim 80$ galaxies.

\section{First results}

A first test on the formation and evolution of elliptical and S0 galaxies is made with the Kormendy relation (KR), a scaling relation between the effective radius $R_{e}$ and effective surface brightness $\langle\mu\rangle_{e}$. As this relation is not based on galaxy kinematics, it can be studied to fainter magnitudes.

The KR for two low $L_{X}$ clusters, $\mathrm{Cl} 0841$ and $\mathrm{Cl} 1701$, is shown in Fig. 1. We compare these poor clusters with two rich clusters, A 2218 and A 2390 at similar redshift $(z \sim 0.2)$ 


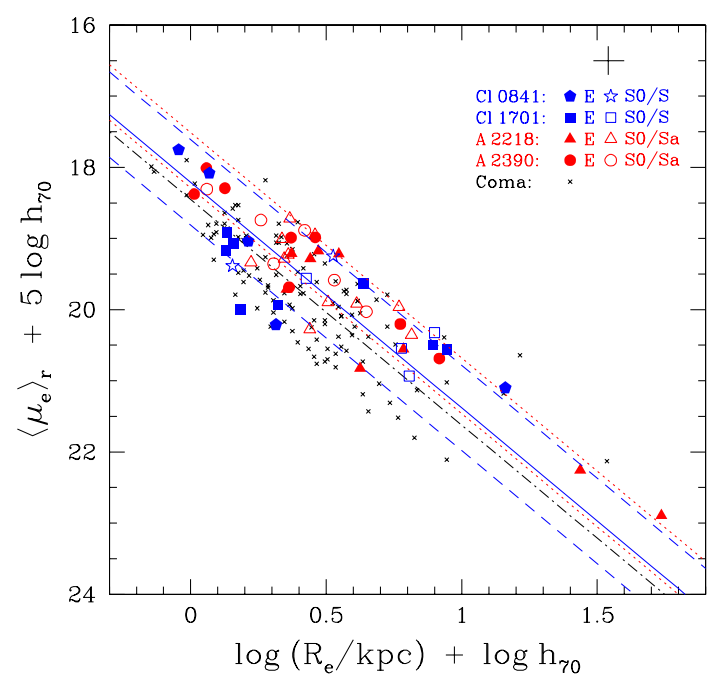

Figure 1. KR in Gunn $r$ for the poor clusters Cl $0841(z=0.24)$ and $\mathrm{Cl} 1701(z=0.25)$, compared to the rich clusters A $2218(z=0.18)$ and A $2390(z=0.23)$ and to the local Coma sample of JFK95. The dot-dashed line is a least square fit to Coma. Dotted lines show the $1 \sigma$ errors of the fit for the rich clusters, the solid line indicates the fit for the poor clusters with $1 \sigma$ (dashed lines), assuming the local slope. A typical error bar is in the upper right corner.

and to the local Coma sample of Jørgensen et al. 1995 (JFK95). In total, the low $L_{X}$ sample comprises $19 \mathrm{E}+\mathrm{S} 0$ galaxies, divided into $13 \mathrm{E}$ and $6 \mathrm{~S} 0$ galaxies. On average, all these objects show an evolution of $\bar{m}_{r} \sim 0.24$ compared to Coma. The 34 rich cluster galaxies are on average brighter by $\sim 0.47 \mathrm{mag}$. The small, not significant, difference might be caused by the fact that the two samples comprise slightly different galaxies in luminosity. However, as the scatter of the low $L_{X}$ galaxies is quite large, a more quantitative analysis must be based on the whole cluster sample.

\section{Acknowledgements}

This project is a collaboration with R. G. Bower, M. L. Balogh and I. Smail (Durham/UK) and R. L. Davies (Oxford/UK) who have contributed to these results. We thank the Calar Alto staff for efficient observational support. AF and BLZ acknowledge financial support by the Volkswagen Foundation (I/76 520) and the DFG (ZI 663/3-1, ZI 663/5-1).

\section{References}

Balogh, M. L., Smail, I., Bower, R. G. 2002a ApJ 566, 123-136.

Balogh, M., Bower, R. G., Smail, I., Ziegler, B. L., Davies, R. L., Gaztelu, A., Fritz, A. 2002b MNRAS 337, 256-274.

Cole, S., Lacey, C. G., Baugh, C. M., Frenk, C. S. 2000 MNRAS 319, 168-204.

Djorgovski, S., Davis, M. 1987 ApJ 313, 59-68.

Fritz, A., Ziegler, B. L., Bower, R. G. et al. $2004 M N R A S$, submitted.

Jørgensen, I., Franx, M., Kjærgaard, P. 1995 MNRAS 273, 1097-1128. (JFK95)

van Dokkum, P. G., Stanford, S. A. 2003 ApJ 585, 78-89.

Ziegler, B. L., Bender, R. 1997 MNRAS 291, 527-543.

Ziegler, B. L., Bower, R. G., Smail, I. et al. 2001 MNRAS 325, 1571-1590. 